\title{
Comprehensive study of various light trapping techniques used for sandwiched thin film solar cell structures
}

S. Abdellatif, K. Kirah, R. Ghannam, A. S. G. Khalil, W. Anis

S. Abdellatif, K. Kirah, R. Ghannam, A. S. G. Khalil, W. Anis, "Comprehensive study of various light trapping techniques used for sandwiched thin film solar cell structures," Proc. SPIE 10527, Physics, Simulation, and Photonic Engineering of Photovoltaic Devices VII, 1052715 (16 February 2018); doi: 10.1117/12.2291613

SPIE. Event: SPIE OPTO, 2018, San Francisco, California, United States 


\title{
Comprehensive study of various light trapping techniques used for sandwiched thin film solar cell structures
}

\author{
S. Abdellatif, ${ }^{1}$ K. Kirah, ${ }^{2, *}$ R. Ghannam, ${ }^{3}$ A. S. G. Khalil, ${ }^{4,5}$ and W. Anis ${ }^{2}$ \\ ${ }^{1}$ The British University in Egypt (BUE), Cairo, Egypt \\ ${ }^{2}$ Faculty of Engineering, Ain Shams University, Cairo, Egypt \\ ${ }^{3}$ School of Engineering, University of Glasgow, United Kingdom \\ ${ }^{4}$ Arab Academy for Science, Technology and Maritime Transport, Giza, Egypt \\ ${ }^{5}$ Physics Department, Faculty of Science, Fayoum University, Fayoum, Egypt \\ *Corresponding author: khaled.kirah@eng.asu.edu.eg
}

\begin{abstract}
Thin film solar cells (TFSCs) where first introduced as a low cost alternative to conventional thick ones. TFSCs show low conversion efficiencies due to the used poor quality materials having weak absorption capabilities and to thin absorption layers. In order to increase light absorption within the active layer, specially near its absorption edge, photon management techniques were proposed. These techniques could be implemented on the top of the active layer to enhance the absorption capabilities and/or to act as anti-reflecting coating structures. When used at the back side, their purpose is to prevent the unabsorbed photons from escaping through the back of the cell.

In this paper, we coupled the finite difference time-domain (FDTD) algorithm for simulating light interaction within the cell with the commercial simulator Comsol Multiphysics 4.3b for describing carrier transports. In order to model the dispersive and absorption properties of various used materials, their complex refractive indices were estimated using the Lorentzian-Drude (LD) coefficients. We have calculated the absorption profile in the different layers of the cell, the external quantum efficiency and the power conversion efficiency achieved by adding dielectric nanospheres on the top of the active layer. Besides that, the enhancement observed after the addition of dielectric nanospheres at the back side of the active layer was computed. The obtained results are finally compared with the effects of using textured surface and nanowires on the top in plus of cascaded 1D and 2D photonic crystals on the back.
\end{abstract}

Keywords: Thin film solar cell, Photon management, Dielectric nanospheres, Photonic crystals

\section{INTRODUCTION}

A thin film solar cell (TFSC) is made by depositing one or more thin layers (thin films) of photovoltaic material on a substrate [1,2]. The thickness range of such layers is wide and varies from a few nanometers to tens of micrometers [3]. TFSC are usually produced by physical or chemical deposition techniques [4], which can be easily applied to large areas and which offers reduced costs. However, TFSCs suffer from low conversion efficiency due to low cost materials (weak absorption capabilities) and thin absorption layers [5]. To enhance the conversion efficiency of thin film solar cells, various optical enhancement techniques have been used [6-11].

We consider a TFSC having amorphous silicon (a-Si) as the active material. The absorption in the TFSC is limited by the absorption capabilities of the active layer in the visible region. Most photons in the near infrared region, above 700 $\mathrm{nm}$, will probably escape from the back side. In order to prevent such escaping, photon management techniques could be used [10,12-16]. These techniques could be implemented on the top of the active layer to enhance the absorption capabilities of the active layer [17-19]. In addition, to prevent the unabsorbed photons escaping from the back side of the cell $[7,20]$, usually a textured back reflector is used to reflect the incoming light at an oblique angle [21]. As long as the light can deviate from the normal direction with an angle larger than the critical angle, total internal reflection can occur at the top surface and the light path can be further increased. This could be done using photonic structures [22] which are 
structures with periodic dielectric constants in one, two or three dimensions with lattice periods approximately equal to the wavelengths of interest. In another light trapping technique, a colloidally synthesized array of resonant dielectric spheres is deposited on the top of the active layer [23]. In the near field, the spheres act as photonic crystal light-trapping elements by coupling light into the absorber layer. In the far-field, a graded index antireflection coating is formed.

In an earlier publication, we simulated an active layer sandwiched between two light trapping structures [16]. The upperside part consisted of an indium tin oxide (ITO) layer which acts as an electrical contact and also as an anti-reflection coating (ARC). An array of a-Si nanowires then follows to enable an efficient and broad band ARC. The back-side part is a cascaded 2D and 1D photonic crystal structures. In the present work, another form of a sandwiched light trapping structure is introduced. Two hexagonally closed packed (HCP) structures of dielectric nanospheres are simulated on the top and on the bottom of the a-Si active layer as shown in figure 1. A comparison between the two sandwiching strategies will conclude this study.

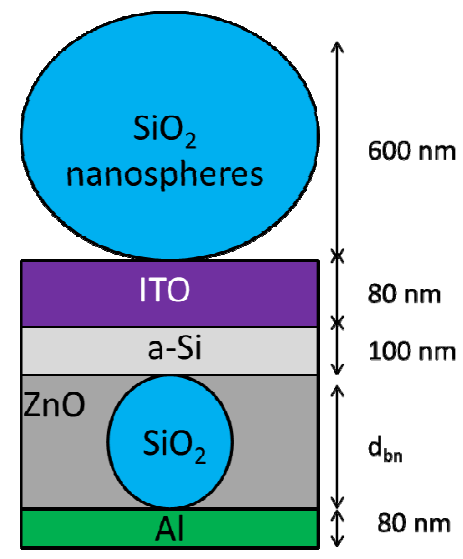

Figure 1. Schematic diagram for TFSC with sandwiched HCP array of nanospheres

\section{2- THE SIMULATED STRUCTURE}

For the top dielectric nanosphere layer, the sphere size is chosen in the order of the peak wavelength (around $600 \mathrm{~nm}$ ). It is shown that whispering gallery modes in the spheres can be coupled into particular modes of the solar cell and significantly enhance its efficiency [23]. Although this front layer enhances the amount of light that reaches the active layer, there is still some parasitic absorption mainly in the back metal reflector. In order to minimize this parasitic absorption, a backside high reflecting and diffracting layer is inserted using the same HCP structures. For the backside layer, the sphere size is chosen so that a reflectance occurs in the NIR region to trap the unabsorbed photons inside the active layer and prevent them from escaping outside the cell. This is done by performing high diffraction orders to increase the probability of total internal reflection inside the active layer as well as utilizing the scattering phenomena to increase the optical path length.

\section{SIMULATION METHOD}

The described structure is simulated using an integrated opto-electrical simulator. The proposed simulation platform gives both optical simulation outcomes, such as the absorption profile, upper surface reflectance and parasitic absorption, as well as electrical outcomes, such as $J-V$ characteristic curves and the external quantum efficiency (EQE). To simulate light interaction with the different layers of the TFSC, we used the finite difference time-domain (FDTD) algorithm [24] through the free open-source algorithm embedded in MEEP [25]. On the other hand, carrier transport modelling is done as a p-i-n structure using Comsol Multiphysics semiconductor module for the 1D drift diffusion modelling [16,26,27]. The absorption and dispersion behaviours of different materials used in the device are modelled based on the Lorentzian-Drude (LD) fitting as already addressed in [28]. The LD model is chosen due to its compatibility with FDTD simulations. The work implemented here is considered as an extension for the previously published work in $[16,28]$, where more information about the opto-electronic model could be found. 


\subsection{Optical modelling}

AM1.5G is assumed with a maximum intensity of $1000 \mathrm{~W} / \mathrm{m}^{2}$ [29]. TE and TM modes are represented by two equal Gaussian light sources with a mean wavelength of $550 \mathrm{~nm}$ and a width from $200 \mathrm{~nm}$ to $1700 \mathrm{~nm}$. The simulated volume is chosen to be $100 \mu \mathrm{m} \times 100 \mu \mathrm{m} \times 100 \mu \mathrm{m}$ with a calculating grid of $10 \mathrm{~nm} \times 10 \mathrm{~nm} \times 10 \mathrm{~nm}$ and the resolution is set to 10 , (i.e. 10 meshes per calculating grid). Concerning the top HCP light trapping layer suggested in figure 1, two optical mechanisms should be associated, the local modes excitation and scattering. In order to study the local modes, the whispering gallery modes (WGM) in the dielectric nanospheres, Maxwell's equations in spherical coordinates are reduced to sets of equations in scalar quantities known as Debye potential [30]. The general form of Maxwell's equations in Gaussian units for a monochromatic field are:

$\nabla \times E=i k H \quad$ and $\quad \nabla \times H=-i k E$

where $E$ and $H$ are the electric field intensity and magnetic field intensity respectively, $i$ is the imaginary unit, $k$ is the wave number $\left(k=\frac{\omega}{c} \sqrt{\mu_{r} \varepsilon_{r}}\right), \omega$ is the angular frequency, $c$ is the speed of light in free space, $\mu_{r}$ and $\varepsilon_{r}$ are the medium relative permeability and relative permittivity respectively. Using the detailed analytical solution given in [30], one can reach the two general transcendental equations for both TE and TM modes inside the nanospheres respectively as follow:

$\frac{\left[\sqrt{k a} J_{v}(k a)\right]^{\prime}}{\sqrt{k a} J_{v}(k a)}=\sqrt{\varepsilon_{r} / \mu_{r}} \frac{\left[\sqrt{k_{o} a} H_{v}^{\{1\}}\left(k_{o} a\right)\right]^{\prime}}{\sqrt{k_{o} a} H_{v}^{\{1\}}\left(k_{o} a\right)}$

$\frac{\left[\sqrt{k a} J_{v}(k a)\right]^{\prime}}{\sqrt{k a} J_{v}(k a)}=\sqrt{\mu_{r} / \varepsilon_{r}} \frac{\left[\sqrt{k_{o} a} H_{v}^{\{1\}}\left(k_{o} a\right)\right]^{\prime}}{\sqrt{k_{o} a} H_{v}^{\{1\}}\left(k_{o} a\right)}$

where $k_{o}=\frac{\omega}{c}$ represents the wave number in air, $a$ is the sphere radius, $H_{v}^{\{1\} v}$ is the Hankel function of the first kind, $J_{v}$ is the Bessel function and the prime sign indicates a derivative with respect to the argument. Herein, two integers $n$ and $m(n \leq m)$ are introduced to describe the arbitrary constants which appear during solving the differential equations. The Bessel and Hankel argument (v) is defined by:

$v=n+\frac{1}{2}$

By solving the two transcendental equations (2 and 3) in terms of $\left(k_{o} a\right)$, a set of roots (eigenfrequencies) could be determined. As these equations have many roots, another index $q$, will be introduced to indicate the order of the root, so that the mode will be described by $n, m, q$. It is important to mention that the transcendental equations are independent on the index $m$. This will lead to degenerate modes across this index.

On the other hand, Comsol Multiphysics is used for modeling the scattering mechanism due to the HCP array of dielectric nanospheres by utilizing the Mie scattering model in [31]. The results assume elastic scattering only and do not include Brillouin or Raman scattering. The solutions provide details of the absorption, scattering, extinction, pressure cross-sections, back-scattering and radiation force exerted on the particle by an incident plane wave. The results are compared with the Mie analytical solution and show good agreement [32].

\subsection{Carrier transport modeling}

Carrier transport in the simulated structure is carried out using the commercial simulator Comsol Multiphysics 5. This version offers a semiconductor material library making it possible to simulate various semiconductor devices governed by the drift-diffusion, Poisson and the continuity equations assuming carrier transport in one direction. Comsol uses finite element method (FEM) solver where a tetrahedral mesh was selected with a tunable size according to the minimum feature length in the TFSC structure. The targeted thin film solar cell is simulated as a $p-i-n$ junction. The surface, the bulk recombinations and the contact effects are also considered. With a doping level of $10^{18} \mathrm{~cm}^{-3}$ in both the $p$ and $n$ sides, the optical generation rate term is extracted from the optical model and the material parameters are used from the Comsol Multiphysics library. 


\section{RESULTS AND DISCUSSIONS}

\subsection{Front-side light trapping structure}

In this section, we introduce the effect of deposing an array of HCP silica nanospheres on the top of a thin film solar cell. The selected reference TFSC, shown in figure 2a, is the same introduced in [16,27] for post comparisons and verification. It is a bare cell without any photon management structure. A layer of $300 \mathrm{~nm}$ radius silica nanospheres is deposited on the top of the TFSC in hexagonally packaged arrangement (shown in figure 2b). As the packing topology affects the coupling effects between coherent spheres, hexagonally close packed spheres causes a diffractive coupling modes which can couple all with each other [33]. For close packed spheres, the distances between the spheres centers are taken to be equal to the sphere diameter. To calculate the values of the eigenwavelengths of the WGM, the procedure introduced in section 3.1 is used. The solution for the electrical field shows interesting resonances at $430 \mathrm{~nm}, 520 \mathrm{~nm}$ and $670 \mathrm{~nm}$ in the spectrum from $300 \mathrm{~nm}$ to $800 \mathrm{~nm}$. This portion matches the solar spectrum range below the cutoff wavelength of a-Si at $800 \mathrm{~nm}$ [34].

(a)

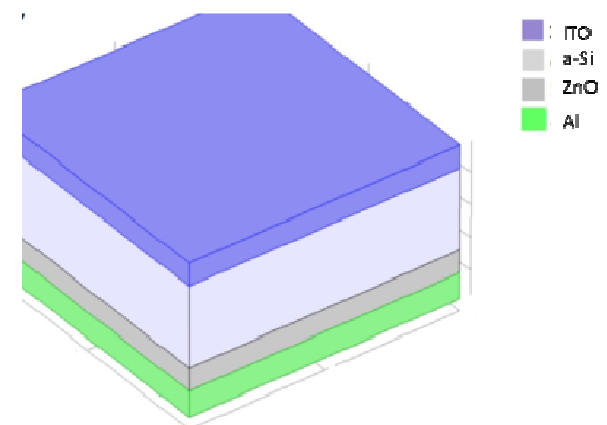

(b)

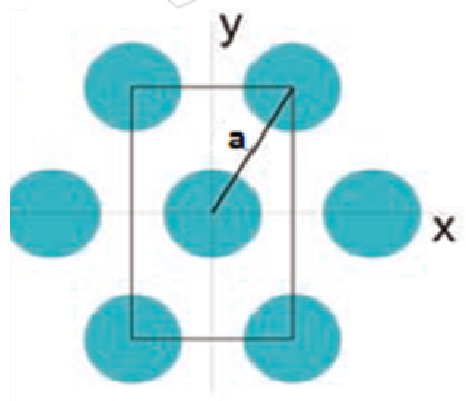

Figure 2. (a) A schematic diagram for the TFSC under test, (b) $\mathrm{HCP} \mathrm{SiO}_{2}$ nanospheres unit cell

A cross-section schematic for the simulated structure in shown in figure $3 \mathrm{a}$, while a cross-sectional plot for the normalized electric field distribution as well as the absorption curve are plotted in figure $3 \mathrm{~b}$. The normalized electric field curve (dotted red curve) shows an oscillating mode inside the silica sphere and another small unwanted propagation inside the aluminium doped $\mathrm{ZnO}$ layer. In addition, an interesting spike at the ITO sphere interface is observed indicating the coupling field from the lower index medium, silica sphere, to the higher index layer (ITO thin film). Besides that, the absorption curve (solid blue curve) shows peak absorption inside the a-Si active layer, with some parasitic absorption inside the aluminium back electrode. How to avoid such parasitic absorption will be addressed in the next section.

(a)

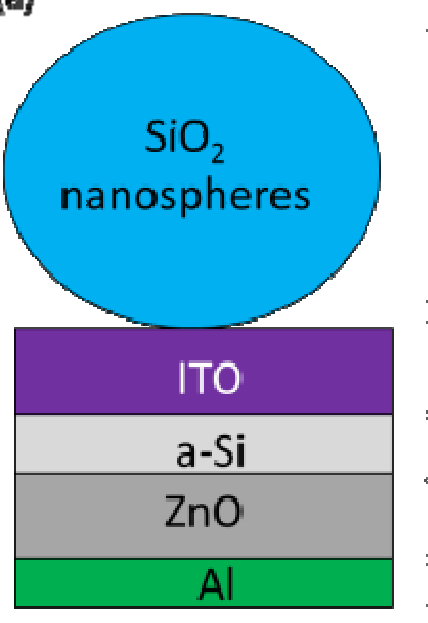

$600 \mathrm{~nm}$

(b)

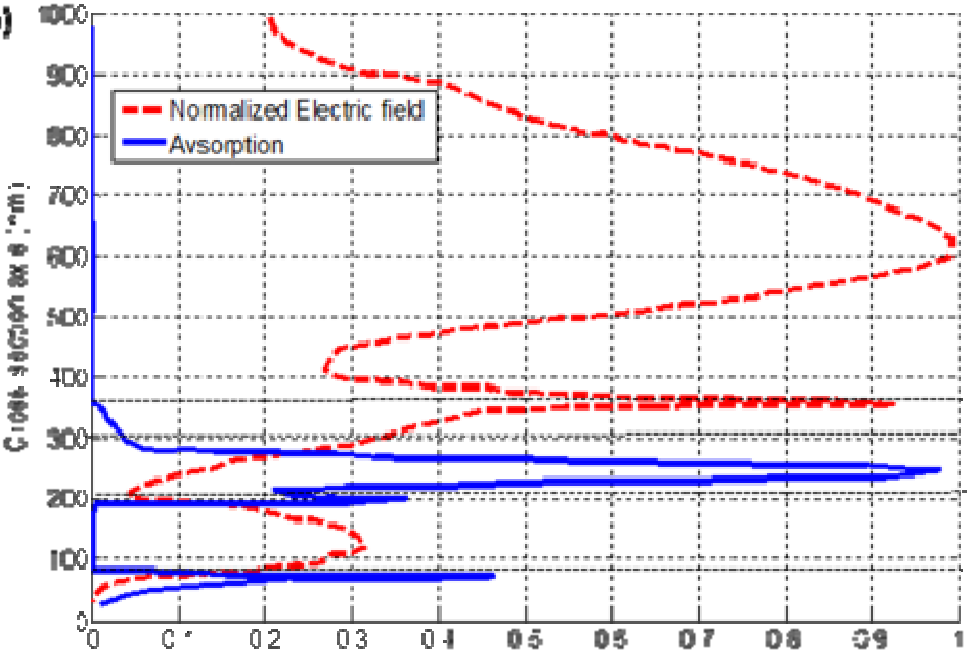

Figure 3. (a) A cross-sectional schematic diagram for the TFSC under test with silica nanosphere, (b) The normalized electric field and absorption across the structure cross section. 
Finally, the electrical parameters are discussed through investigating the external quantum efficiency (EQE) and the $J-V$ characteristic curve for both with and without the silica spheres as shown in figure 4(a) and 4(b). The electrical $p-i-n$ model, used in modelling the electrical behaviour of TFSC which is already introduced in section 3.2 is used for calculating the electrical parameters for this structure as well. The EQE curves reflect a significant enhancement in the $\mathrm{EQE}$ by about $20 \%$ and $12 \%$ for wavelengths $680 \mathrm{~nm}$ and $520 \mathrm{~nm}$ respectively. On the other hand, the $J-V$ characteristic curve for the TFSC with HCP nanospheres shows an enhancement in short circuit current over that of the flat TFSC by around $14.8 \%$ (from $8.1 \mathrm{~mA} / \mathrm{cm}^{2}$ to $9.3 \mathrm{~mA} / \mathrm{cm}^{2}$ ), reflecting on the total conversion efficiency by $9 . \%$ (from $4.4 \%$ to $4.8 \%)$.

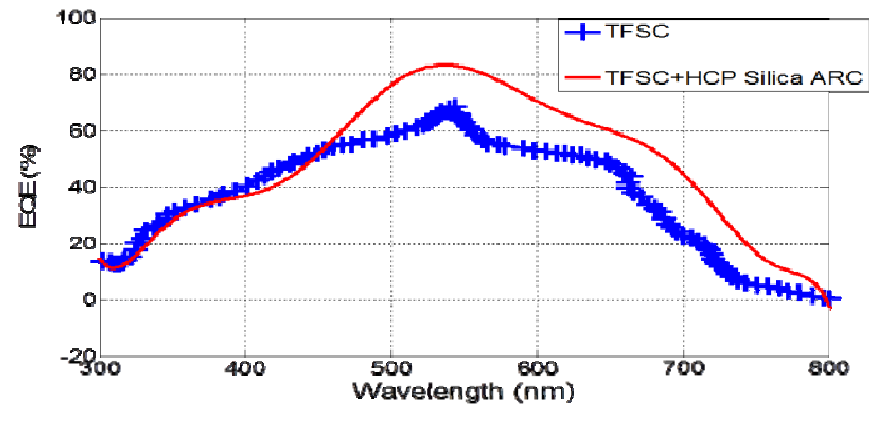

(a)

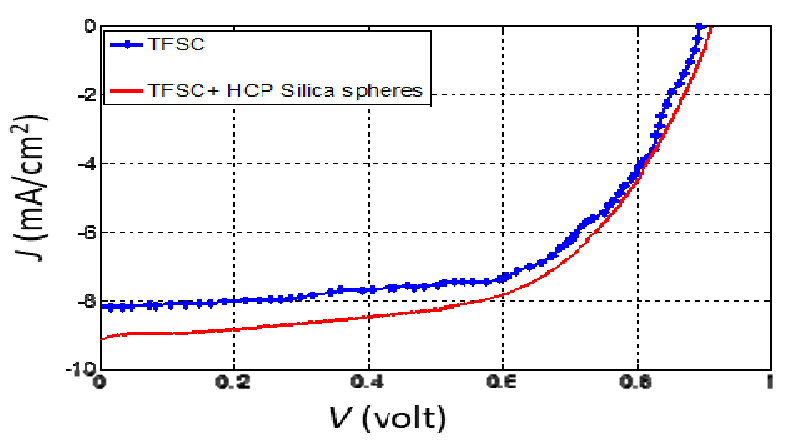

(b)

Figure 4: (a) The EQE and (b) the $J-V$ characteristic curve for a TFSC with and without HCP silica spheres

\subsection{Back-side light trapping structure}

Figure 3 is showing some parasitic absorption located in the metal back reflector layer. This absorption is attributed to escaping photons that are not well trapped inside the active layer. Therefore, a more powerful trapping structure is required. A cascaded back structure is implemented by adding a second diffracting layer of HCP silica spheres inside the $\mathrm{ZnO}$ back reflector. The added layer should behave as high diffracting structures, reducing the probability of escaping photons. As theses spheres should be in sub-wavelength scale, and in order to maintain the same active layer thickness, the $\mathrm{ZnO}$ layer thickness will vary to adapt the back-side spheres. For optimum back-side diffracting layer, the $\mathrm{HCP} \mathrm{SiO}_{2}$ sphere diameters should be optimized for maximum conversion efficiency. Accordingly, the back-side sphere diameter versus efficiency is plotted in figure 5. The figure shows a maximum conversion efficiency of $5.12 \%$ at a diameter of $240 \mathrm{~nm}$. This is where the efficiency starts to decreases by increasing the diameter from the sub-wavelength region to the wavelength region, as the photonic crystal capabilities starts to deteriorate. To examine the utility of the optimized backside diffracting layer, the absorption across the structure at the coupling wavelength, $\lambda=680 \mathrm{~nm}$ is plotted in figure 6 showing the reduction in the parasitic absorption with respect to that without diffracting layer. Finally, figure 7 shows the effect of the different proposed sandwiched structures on the $J-V$ curves. The structure with front and back nanospheres shows an enhancement in the short circuit current, reaching $9.7 \mathrm{~mA} / \mathrm{cm}^{2}$. This enhancement in short circuit current results in an overall enhancement by around $19.7 \%$ with respect to the reference cell. 


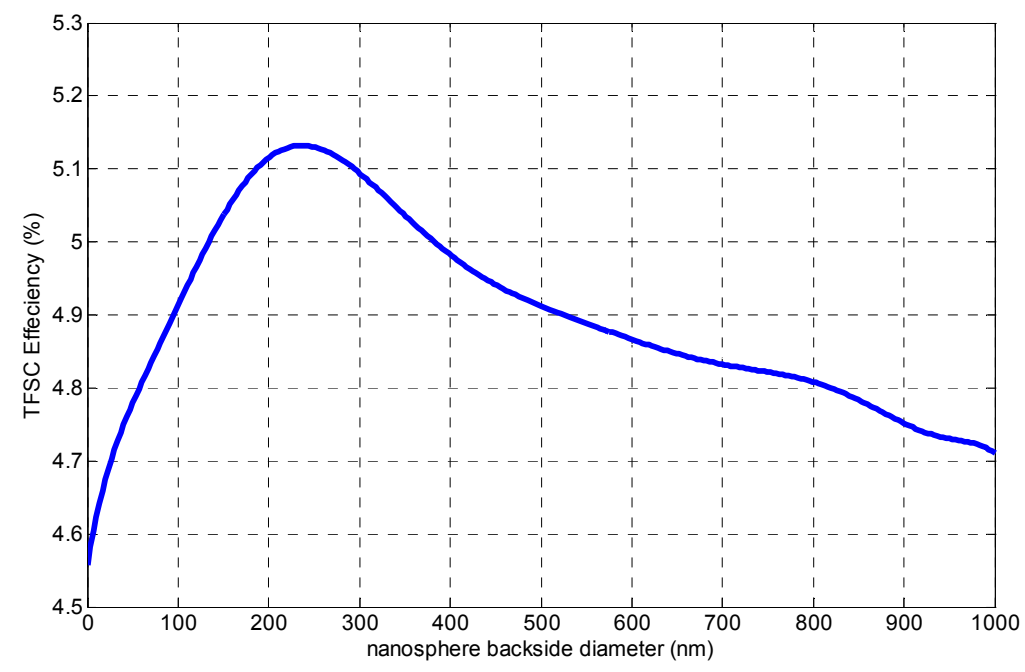

Figure 5. Optimizing back-side spheres radius for maximum conversion efficiency.

It is worth to mention that one of the advantages of this structure is in the experimental applicability and relatively low cost deposition techniques that can be utilized. The deposition of the HCP array of silica spheres either on BK7 glass, FTO coated glass or silicon using spin coating self-assembly deposition are addressed in the literature [35,36]. The deposited arrays showed acceptable stability leading to stable enhanced TFSC.

(a)

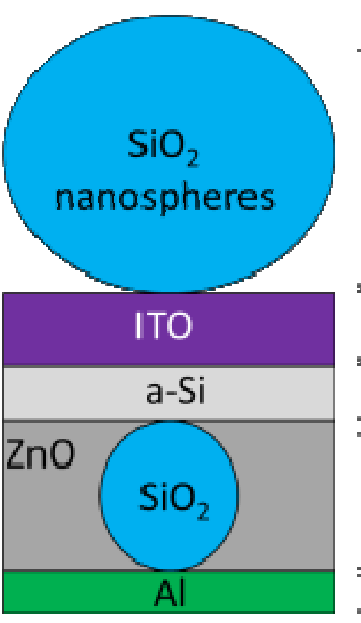

(b)

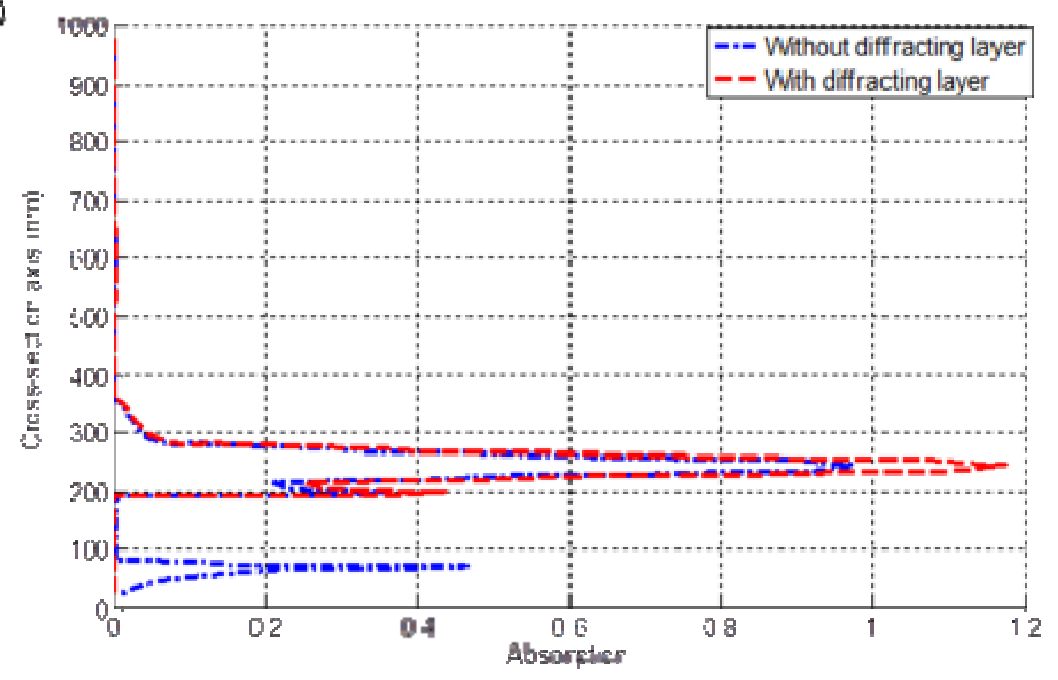

Figure 6. The enhancement in absorption due to the back-side diffracting layer. 


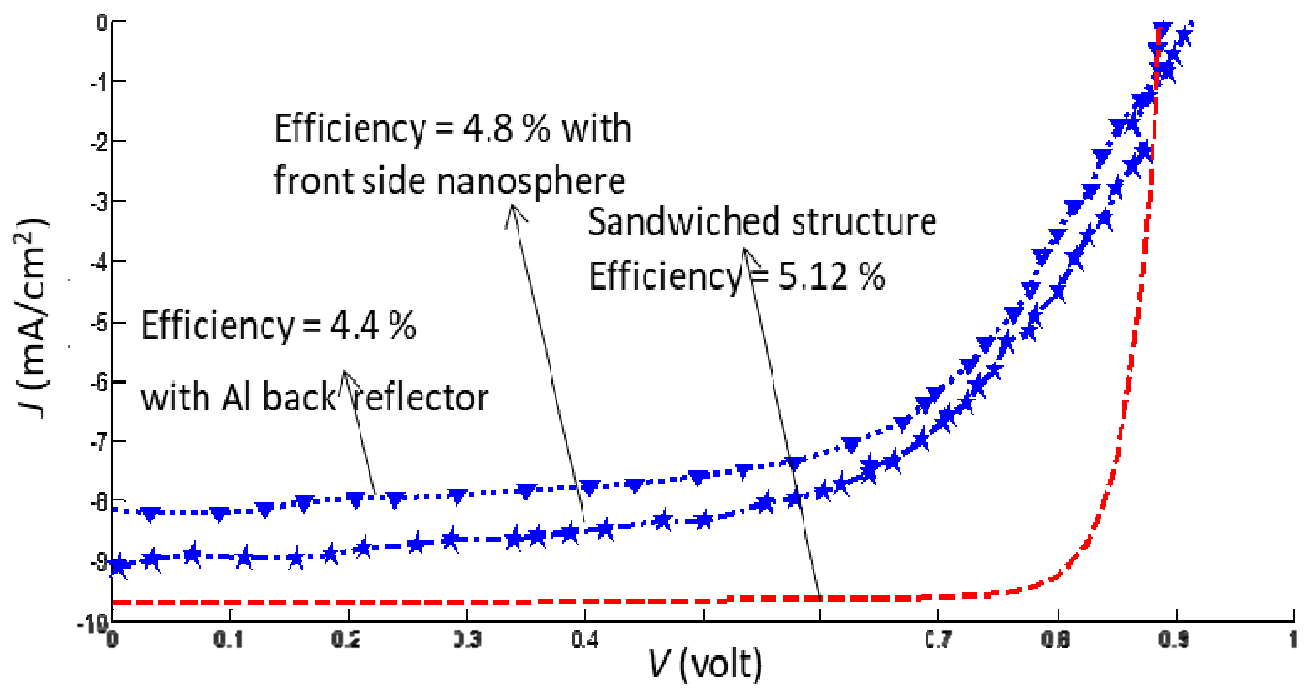

Figure 7. The $J-V$ curve for TFSC under three different proposed structures for light trapping.

\subsection{Comparison of the different sandwiched geometries}

Table 1 shows a comparison between the results obtained in this study and those obtained in [16].

Table 1: Comparison between results obtained with several sandwiched geometries

\begin{tabular}{|l|c|c|c|c|}
\hline \multicolumn{1}{|c|}{ Geometry } & $\begin{array}{c}J_{S C} \\
\left(\mathrm{~mA} / \mathrm{cm}^{2}\right)\end{array}$ & $\begin{array}{c}\% \\
\text { enhancement }\end{array}$ & $\begin{array}{c}\text { Power Conversion } \\
\text { Efficiency \% }\end{array}$ & $\begin{array}{c}\% \\
\text { enhancement }\end{array}$ \\
\hline Bare cell_1 & 8.1 & ----- & 4.4 & ----- \\
\hline Cell_1 with nanosphere on the top & 9.3 & 14.8 & 4.8 & 9.0 \\
\hline $\begin{array}{l}\text { Cell_1 with nanosphere on the top } \\
\text { and on the back }\end{array}$ & 9.7 & 19.7 & 5.12 & 16.3 \\
\hline Bare cell_2 & 8.1 & ----- & 4.12 & ----- \\
\hline Cell_2 with PCs on the back & 9.6 & 18.5 & 5.12 & 24.7 \\
\hline $\begin{array}{l}\text { Cell_2 with PCs on the back and } \\
\text { textured surface on the top }\end{array}$ & 10.1 & 24.7 & 5.23 & 26.9 \\
\hline $\begin{array}{l}\text { Cell_2 with PCs on the back, } \\
\text { textured surface and NWs on the top }\end{array}$ & 11.0 & 35.8 & 6.03 & 46.3 \\
\hline
\end{tabular}

The table shows better enhancement in the case of using PCs on the back and textured surface and NWs on the top. However, the realization of such structure is complicated and consequently more expensive. On the other hand, even if it gives lower figures, the fabrication of the nanosphere is simpler and low cost, which is certainly a clear advantage.

\section{CONCLUSION}

A novel structure combining a top and a back HCP arrays of dielectric nanospheres is simulated. Above the active layer, the nanospheres acts as an ARC as well as coupling medium by exciting whispering gallery modes at selected wavelengths. Below the active layer, the nanospheres diffracts the incoming light over the standard $\mathrm{ZnO} / \mathrm{Al}$ reflector. We have calculated the external quantum efficiency and the power conversion efficiency of the studied structures. The obtained results are compared to the results for another geometry consisting of PCs on the back, textured surface and NWs on the top. The nanosphere solution seems to be a good compromise between lower fabrication costs and an acceptable increase in efficiency. 


\section{ACKNOWLEDGEMENT}

This work is part of SURSYS project funded by the German Academic Exchange Service (DAAD) and financed by the Federal Foreign Office. The authors acknowledge the valuable support of Prof. Mathias Ulbricht, Dr. Michael Eisinger and Mr. Simon Kustos (Kresmann). We would also like to acknowledge the support of The World Academy of Sciences (TWAS) for providing the necessary computational resources.

\section{REFERENCES AND LINKS}

[1] A. G. Aberle, "Thin-film solar cells," Thin Solid Films, vol. 517, pp. 4706-4710 (2009).

[2] R. B. Bergmann, "Crystalline Si thin-film solar cells: a review," Applied Physics A, vol. 69, pp. 187-194 (1999)

[3] K. Chopra and S. Das, "Why Thin Film Solar Cells?," Thin Film Solar Cells: Springer US, pp. 1-18 (1983).

[4] A. Shah, P. Torres, R. Tscharner, N. Wyrsch, and H. Keppner, "Photovoltaic Technology: The Case for Thin-Film Solar Cells," Science, vol. 285, pp. 692-698 (1999)

[5] M. A. Green, K. Emery, Y. Hishikawa, W. Warta, and E. D. Dunlop, "Solar cell efficiency tables (Version 45)," Progress in Photovoltaics: Research and Applications, vol. 23, pp. 1-9 (2015).

[6] J. Müller, B. Rech, J. Springer, and M. Vanecek, "TCO and light trapping in silicon thin film solar cells," Solar Energy, vol. 77, pp. 917-930 (2004).

[7] D. Zhou and R. Biswas, "Photonic crystal enhanced light-trapping in thin film solar cells," Journal of Applied Physics, vol. 103, pp. 093102 (2008).

[8] C. Haase and H. Stiebig, "Thin-film silicon solar cells with efficient periodic light trapping texture," Applied Physics Letters, vol. 91, pp. 061116 (2007).

[9] S.-B. Rim, S. Zhao, S. R. Scully, M. D. McGehee, and P. Peumans, "An effective light trapping configuration for thin-film solar cells," Applied Physics Letters, vol. 91, pp. 243501 (2007).

[10] C. S. Schuster, "Nanostructures for Enhanced Light-Trapping in Thin-Film Silicon Solar Cells," in Diffractive Optics for Thin-Film Silicon Solar Cells: Springer, pp. 11-51 (2017).

[11] V. Jovanov, E. Moulin, F.-J. Haug, A. Tamang, S. I. H. Bali, C. Ballif, D. Knipp, "From randomly self-textured substrates to highly efficient thin film solar cells: Influence of geometric interface engineering on light trapping, plasmonic losses and charge extraction," Solar Energy Materials and Solar Cells, vol. 160, pp. 141-148 (2017).

[12] C. Rockstuhl, T. Paul, C. Menzel, K. Bittkau, T. Beckers, R. Carius, and F. Lederer, "Photon Management in Thin Film Solar Cells," presented at the International Quantum Electronics Conference Baltimore, Maryland United States, (2009).

[13] C. Rockstuhl and F. Lederer, "Photon management by metallic nanodiscs in thin film solar cells," Applied Physics Letters, vol. 94, p. 213102 (2009).

[14] W. Wang, S. Wu, K. Reinhardt, Y. Lu, and S. Chen, "Broadband Light Absorption Enhancement in Thin-Film Silicon Solar Cells," Nano Letters, vol. 10, pp. 2012-2018 (2010).

[15] S. F. C. Rockstuhl, K. Bittkau, T. Beckers, R. Carius, F.-J. Haug, T. Söderström, C. Ballif, and F. Lederer,

"Comparison and optimization of randomly textured surfaces in thin-film solar cells," Optics Express vol. 18, p. A335 (2010).

[16] S. Abdellatif, K. Kirah, R. Ghannam, A. Khalil, and W. Anis, "Enhancing the absorption capabilities of thin-film solar cells using sandwiched light trapping structures," Applied optics, vol. 54, pp. 5534-5541 (2015).

[17] M. Berginski, J. Hüpkes, M. Schulte, G. Schöpe, H. Stiebig, and B. Rech"The effect of front ZnO:Al surface texture and optical transparency on efficient light trapping in silicon thin-film solar cells," Journal of Applied Physics, vol. 101, p. 074903 (2007).

[18] J.-Y. Jung, H.-D. Um, S.-W. Jee, K.-T. Park, J. H. Bang, and J.-H. Lee, "Optimal design for antireflective Si nanowire solar cells," Solar Energy Materials and Solar Cells, vol. 112, pp. 84-90 (2013).

[19] C. Chen, R. Jia, H. Yue, H. Li, X. Liu, D. Wu, W. Ding, T. Ye, S. Kasai, H. Tamotsu, J. Chu, and S. Wang, "Silicon nanowire-array-textured solar cells for photovoltaic application," Journal of Applied Physics, vol. 108, p. 094318 (2010).

[20] J. Krc, M. Zeman, S. L. Luxembourg, and M. Topic, "Modulated photonic-crystal structures as broadband back reflectors in thin-film solar cells," Applied Physics Letters, vol. 94, p. 153501 (2009).

[21] J. Gjessing, A. S. Sudbø, and E. S. Marstein, "Comparison of periodic light-trapping structures in thin crystalline silicon solar cells," Journal of Applied Physics, vol. 110, p. 033104 (2011). 
[22] J. D. Joannopoulos, P. R. Villeneuve, and S. Fan, "Photonic crystals: putting a new twist on light," Nature, 10.1038/386143a0 vol. 386, pp. 143-149 (1997).

[23] J. Grandidier, M. G. Deceglie, D. M. Callahan, and H. A. Atwater, "Simulations of solar cell absorption enhancement using resonant modes of a nanosphere array," Journal of Photonics for Energy, vol. 2, pp. 024502 (2012). [24] K. S. Yee and J. S. Chen, "The finite-difference time-domain (FDTD) and the finite-volume time-domain (FVTD) methods in solving Maxwell's equations," IEEE Transactions on Antennas and Propagation, vol. 45, pp. 354-363 (1997). [25] A. F. Oskooi, D. Roundy, M. Ibanescu, P. Bermel, J. D. Joannopoulos, and S. G. Johnson, "MEEP: A flexible freesoftware package for electromagnetic simulations by the FDTD method," Computer Physics Communications, vol. 181, pp. 687-702 (2010).

[26] Comsol Multiphysics, "version 4.3," Heat Transfer Module.

[27] S. Abdellatif and K. Kirah, "Numerical modeling and simulation for a radial pin nanowire photovoltaic device," Energy Procedia, vol. 36, pp. 488-491 (2013).

[28] S. Abdellatif, R. Ghannam, and A. Khalil, "Simulating the dispersive behavior of semiconductors using the Lorentzian-Drude model for photovoltaic devices," Applied optics, vol. 53, pp. 3294-3300 (2014).

[29] C. Gueymard, D. Myers, and K. Emery, "Proposed reference irradiance spectra for solar energy systems testing," Solar energy, vol. 73, pp. 443-467 (2002).

[30] A. N. Oraevsky, "Whispering-gallery waves," Quantum Electronics, vol. 32, pp. 377-400 (2002).

[31] S. Yushanov, J. S. Crompton, and K. C. Koppenhoefer, "Mie scattering of electromagnetic waves," in Proc. COMSOL conf., pp. 1-7 (2013).

[32] C. Mätzler, "MATLAB functions for Mie scattering and absorption, version 2," IAP Res. Rep, vol. 8, pp. 1-24, (2002).

[33] X. Yu, L. Shi, D. Han, J. Zi, and P. V. Braun, "High quality factor metallodielectric hybrid plasmonic-photonic crystals," Advanced Functional Materials, vol. 20, pp. 1910-1916 (2010).

[34] R. Swanepoel, "Determination of the thickness and optical constants of amorphous silicon," Journal of Physics E: Scientific Instruments, vol. 16,p. 1214 (1983).

[35] M. U. Khan, J. Justice, A. Boersma, M. Mourad, R. Ee, A. Blaaderen, J. Wijnhoven, B. Corbett, "Development of photonic crystal structures for on-board optical communication," in SPIE Photonics Europe, pp. 912705-912705-9 (2014).

[36] X. Yan, J. Yao, G. Lu, X. Li, J. Zhang, K. Han, and B. Yang, "Fabrication of non-close-packed arrays of colloidal spheres by soft lithography," Journal of the American Chemical Society, vol. 127, pp. 7688-7689 (2005). 